\title{
A texture component model for anisotropic polycrystal plasticity
}

\author{
T. Böhlke *, G. Risy, A. Bertram \\ Institut für Mechanik, Otto-von-Guericke-Universität Magdeburg, PSF 4120, D-39016 Magdeburg, Germany
}

\begin{abstract}
There are many crystallographic textures which can be approximated by a small number of texture components [see, e.g., Int. J. Mech. Sci. 31(7) (1989) 549]. In some cases, such texture components can be described by central distributions. Central distributions are characterized by a mean orientation and a half width. The classical Taylor model for viscoplastic polycrystals assumes that a discrete set of single crystals deforms homogeneously. If the viscoplastic version of the Taylor model is numerically implemented then the crystallite orientation distribution function (codf) is usually discretized by a set of Dirac distributions, where each of the Dirac distributions represents a single crystal. Due to the specific discretization of the codf this approach requires usually a large number of discrete crystal orientations even if the texture can be described by a small number of texture components. In the present work, we consider face-centered cubic (fcc) polycrystals and compare the classical upper bound model with an approach based on texture components. The texture components are modeled by Mises-Fischer distributions, which are central distributions. The stress of the polycrystal is obtained by a numerical integration of the single crystal stress state over the orientation space.
\end{abstract}

(C) 2004 Elsevier B.V. All rights reserved.

Keywords: Anisotropic viscoplasticity; Crystallographic orientation distribution function; Crystallographic texture; Texture components; Upper bound model

\section{Introduction}

If the distribution of crystal orientations is inhomogeneous in a polycrystal, then the elastic

\footnotetext{
* Corresponding author. Tel.: +49 39167 12592; fax: +49 3916712863.

E-mail address: boehlke@mb.uni-magdeburg.de (T. Böhlke).
}

and the viscoplastic behavior as well as the non-mechanical properties of the polycrystal are generally anisotropic. Such anisotropies have a significant technological importance since they can positively and negatively affect the mechanical properties of the final product.

In many cases crystallographic textures can be described by a rather small number of texture components, e.g. [27,10]. Such texture components can be modeled by central or noncentral

0927-0256/\$ - see front matter (C) 2004 Elsevier B.V. All rights reserved.

doi:10.1016/j.commatsci.2004.09.040 
distributions [8]. An example of a central distribution is the Mises-Fischer distribution on $\mathrm{SO}(3)$ [17], which has similar properties as the normal distribution in $\mathbb{R}^{n}$.

The classical Taylor(-Bishop-Hill) model assumes that a polycrystal deforms homogeneously. Such an approach ensures the fulfillment of the compatibility condition through the aggregate but violates the equilibrium condition on grain boundaries. The Taylor model usually gives reasonable approximations of deformation textures in aluminum and copper but fails to predict the texture formation, e.g., in brass.

If the viscoplastic version of the Taylor model is implemented usually the crystallite orientation distribution function is discretized by a set of Dirac distributions, where each distribution represents a single crystal. This implies that the stress state of the polycrystal is equal to the arithmetic mean of the stress states in the single crystals.

A disadvantage of an approach based on Dirac distributions is that usually a large number (approximately 500-1000) of discrete crystal orientations is required for a precise prediction of the effective mechanical properties. This is also the case if the texture can be described by a small number of texture components. Therefore, due to the high numerical costs an application of the Taylor model at integration points in finite element codes is of limited applicability. In the present work we compare the classical Taylor model based on Dirac distributions with an approach based on texture components which are described by Mises-Fischer distributions. It will be shown that such an approach allows to reduce the number of crystal orientations significantly. In contrast to other texture component models $[28,22]$, the present approach takes into account the half-width of the texture components during the computation of the stresses.

The outline of the paper is as follows: In Section 2 we summerize the constitutive equations applied on the grain scale to model the elasto-viscoplastic behavior of fcc single crystals. In Section 3 the codf is approximated by a set of Mises-Fischer distributions, which are specified by a mean crystal orientation and a half-width describing the scattering of crystals around the mean orientation. Fur- thermore, the effective stress state is specified in terms of an integral of the single crystalline stress state over the orientation space. Numerical examples concerning the representation of the codf by Mises-Fischer distribution functions are presented in Section 4. Special emphasis is given to the modeling of the $R$ value (Lankford coefficient) and the yield stress in textured polycrystals. It is shown that the suggested texture component model drastically reduces the number of degrees of freedom which are necessary to describe crystallographic textures and to determine the effective mechanical properties.

Notation. Throughout the text a direct tensor notation is preferred. The scalar product and the dyadic product are denoted by $\boldsymbol{A} \cdot \boldsymbol{B}=\operatorname{tr}\left(\boldsymbol{A}^{\top} \boldsymbol{B}\right)$ and $\boldsymbol{A} \otimes \boldsymbol{B}$. Symmetric and traceless tensors are designated by a prime, e.g., $\boldsymbol{A}^{\prime}$. The symmetric and the skew part of a 2 nd-order tensor $\boldsymbol{A}$ are denoted by $\operatorname{sym}(\boldsymbol{A})$ and $\operatorname{skw}(\boldsymbol{A})$, respectively. The set of proper orthogonal tensors is specified by $\mathrm{SO}(3)$.

\section{Constitutive modeling on the microscale}

\subsection{Elastic law}

In the following we adopt the assumption that dilatations are purely elastic whereas distortions are purely viscoplastic. Both deformation modes are assumed to be decoupled. The spherical part and the deviatoric part of the Kirchhoff stress tensor $\tau$ are denoted by $\tau^{\circ}$ and $\tau^{\prime}$, respectively. The first is associated with volume changes and the latter with shape changes. The spherical and the deviatoric part of the stress tensor represent the equilibrium part and the dynamic part of the stress tensor, respectively, see e.g., [26].

Due to the aforementioned assumptions, the strain energy density specifying the equilibrium part of the stress tensor depends only on the determinant $J$ of the deformation gradient. The following form of the strain energy density is adopted

$W(J)=\frac{K}{4}\left(J^{2}-2 \ln (J)-1\right)$, 
where $K$ denotes the bulk modulus [21,25]. As a result, the equilibrium stress is given by

$\tau^{\circ}=J \frac{\partial W(J)}{\partial J} \boldsymbol{I}=\frac{K}{2}\left(J^{2}-1\right) \boldsymbol{I}$.

\subsection{Flow rule and lattice spin}

The viscoplastic distortions are considered to result from inelastic deformations in slip systems. The slip rate is assumed to be driven by the resolved shear stress in the corresponding slip system. Therefore, it depends only on the deviatoric part of the stress tensor, which here is equal to the dynamic part of the stress tensor. Distortions of viscoplastic single crystals can be modeled by the following set of equations

$$
\begin{aligned}
\mathbf{0} & =\boldsymbol{D}^{\prime}-\boldsymbol{Q} \operatorname{sym}\left(\widetilde{\boldsymbol{K}}\left(\boldsymbol{Q}^{\top} \tau^{\prime} \boldsymbol{Q}, \tau_{\alpha}^{\mathrm{C}}\right)\right) \boldsymbol{Q}^{\top}, \\
\dot{\boldsymbol{Q}} \boldsymbol{Q}^{-1} & =\boldsymbol{W}-\boldsymbol{Q} \operatorname{skw}\left(\widetilde{\boldsymbol{K}}\left(\boldsymbol{Q}^{\top} \tau^{\prime} \boldsymbol{Q}, \tau_{\alpha}^{\mathrm{C}}\right)\right) \boldsymbol{Q}^{\top},
\end{aligned}
$$

see, e.g., [11]. $\boldsymbol{D}^{\prime}$ and $\boldsymbol{W}$ are the traceless symmetric and the skew part of the velocity gradient $L=\partial \boldsymbol{v} /$ $\partial \boldsymbol{x}$. The internal variables $\tau_{\alpha}^{\mathrm{C}}$ are the critical resolved shear stresses in the different slip systems. In the present work, fcc single crystals are considered. For this specific class of materials, it is a reasonable assumption that the slip systems harden in an isotropic manner, i.e. $\tau_{\alpha}^{\mathrm{C}}=\tau^{\mathrm{C}}[13]$.

An orthogonal tensor $\boldsymbol{Q}$ is used in order to specify the single crystal orientation. $\boldsymbol{Q}$ is introduced in such a way that it maps a reference basis $\boldsymbol{e}_{i}$ onto the lattice vectors $\boldsymbol{g}_{i}$ at time $t \geqslant 0: \boldsymbol{g}_{i}(t)=\boldsymbol{Q}(t) \boldsymbol{e}_{i}$. If the two sets of lattice vectors $\boldsymbol{e}_{i}$ and $\boldsymbol{g}_{i}(t)$ are known, the orthogonal tensor $\boldsymbol{Q}$ can be computed by $\boldsymbol{Q}=\boldsymbol{g}_{i} \otimes \boldsymbol{e}_{i}$. For given strain rate tensor $\boldsymbol{D}^{\prime}$ and crystal orientation $\boldsymbol{Q}$, Eq. (3) ${ }_{1}$ is an implicit equation for the stress deviator $\tau^{\prime}$. For given $\tau^{\prime}, \boldsymbol{W}$ and $\boldsymbol{Q}$, respectively, Eq. (3) $)_{2}$ determines the spin $\dot{\boldsymbol{Q}} \boldsymbol{Q}^{-1}$ of the crystal lattice.

The function $\widetilde{\boldsymbol{K}}$ is assumed to be given by

$$
\begin{aligned}
& \widetilde{\boldsymbol{K}}\left(\boldsymbol{Q}^{\top} \boldsymbol{\tau}^{\prime} \boldsymbol{Q}, \tau^{\mathrm{C}}\right)=\sum_{\alpha=1}^{N} \dot{\gamma}_{\alpha}\left(\tau_{\alpha}, \tau^{\mathrm{C}}\right) \widetilde{\boldsymbol{M}}_{\alpha}, \\
& \dot{\gamma}_{\alpha}\left(\tau_{\alpha}, \tau^{\mathrm{C}}\right)=\dot{\gamma}_{0} \operatorname{sign}\left(\tau_{\alpha}\right)\left|\frac{\tau_{\alpha}}{\tau^{\mathrm{C}}}\right|^{m}, \\
& \tau_{\alpha}=\left(\boldsymbol{Q}^{\top} \boldsymbol{\tau}^{\prime} \boldsymbol{Q}\right) \cdot \widetilde{\boldsymbol{M}}_{\alpha}
\end{aligned}
$$

[11]. The Schmid or slip system tensors $\widetilde{\boldsymbol{M}}_{\alpha}=\tilde{\boldsymbol{d}}_{\alpha} \otimes \tilde{\boldsymbol{n}}^{\alpha}$ are rank-one tensors, which are defined in terms of the slip directions $\tilde{\boldsymbol{d}}_{\alpha}$ and the slip plane normals $\tilde{\boldsymbol{n}}^{\alpha}$. In the case of an fcc single crystal at room temperature, the octahedral slip systems $\langle 110\rangle\{111\}$ have to be taken into account $(N=12) . \tau_{\alpha}$ is the resolved shear stress in the slip system $\alpha$. The material parameter $m$ quantifies the strain rate sensitivity of the material. It is generally temperature-dependent and can be estimated by strain rate jump experiments. At room temperature $m$ is usually in the range 50-250. In the limit $m \rightarrow \infty$ a rate-independent behavior is obtained. Note, that Eq. (4) implies that the rate of deformation is positively homogeneous of degree $m$ in the stress tensor, whereas the stress is homogeneous of degree $1 / \mathrm{m}$ in the rate of deformation.

\subsection{Hardening: the Kocks-Mecking model}

The critical resolved shear stress is usually related to the mean dislocation density in the crystal lattice by

$\tau^{\mathrm{C}}(\rho)=\alpha b G \sqrt{\rho}$,

where $G$ is the shear modulus and $b$ is the magnitude of the Burgers vector, respectively. The scalar $\alpha$ is generally only weakly temperature- and strain rate dependent and is considered constant here. The Kocks-Mecking model describes the rate of change of the mean dislocation density in fcc single crystals in the hardening stages II and III, respectively, over a wide range of strain rates and temperatures $[12,9,13]$. In the context of finite deformations the model is given by the following set of equations

$\dot{\rho}\left(\tau_{\alpha}, \rho\right)=\left(\frac{\sqrt{\rho}}{\beta b}-\kappa\left|\frac{\dot{\gamma}\left(\tau_{\alpha}, \rho\right)}{\dot{\gamma}_{0}^{*}}\right|^{-\frac{1}{n}} \rho\right) \dot{\gamma}\left(\tau_{\alpha}, \rho\right)$,

where

$\dot{\gamma}\left(\tau_{\alpha}, \rho\right)=\sum_{\alpha=1}^{N}\left|\dot{\gamma}_{\alpha}\left(\tau_{\alpha}, \tau^{\mathrm{C}}(\rho)\right)\right|$.

The first term in Eq. (8) describes the statistical storage of dislocations (hardening stage II). The second term in (8) models the dynamic recovery 
(hardening stage III), which is strain rate and temperature dependent. A combination of (7) and (8) allows to derive the equivalent formulation of the Kocks-Mecking model in terms of the critical resolved shear stress

$\dot{\tau}^{\mathrm{C}}\left(\tau_{\alpha}, \tau^{\mathrm{C}}\right)=\Theta_{0}\left(1-\frac{\tau^{\mathrm{C}}}{\tau_{\mathrm{V}}^{\mathrm{C}}\left(\tau_{\alpha}, \tau^{\mathrm{C}}\right)}\right) \dot{\gamma}\left(\tau_{\alpha}, \tau^{\mathrm{C}}\right)$,

where the critical Voce stress is

$\tau_{\mathrm{V}}^{\mathrm{C}}\left(\tau_{\alpha}, \tau^{\mathrm{C}}\right)=\tau_{\mathrm{V} 0}^{\mathrm{C}}\left|\frac{\dot{\gamma}\left(\tau_{\alpha}, \tau^{\mathrm{C}}\right)}{\dot{\gamma}_{0}^{*}}\right|^{1 / n}$,

$\Theta_{0}=\alpha G /(2 \beta)$ and $\tau_{\mathrm{V} 0}^{\mathrm{C}}=\alpha G /(\kappa \beta)$. The material parameters have been determined based on experimental data documented by Brandes and Brook [4], Les et al. [16], and Mecking [20], respectively: $K=71.7 \mathrm{GPa}, \quad \dot{\gamma}_{0}=9 \times 10^{-3} \mathrm{~s}^{-1}, \quad m=133, \quad \tau_{0}^{\mathrm{C}}=$ $15 \mathrm{MPa}$ (initial value of the critical Schmid stress), $\Theta_{0}=37.5 \mathrm{MPa}, \tau_{\mathrm{V} 0}^{\mathrm{C}}=51.5 \mathrm{MPa}, n=22.5$ and $\dot{\gamma}_{0}^{*}=$ $10^{7} \mathrm{~s}^{-1}$.

\section{Constitutive equations on the macroscale}

\subsection{Homogenization of the stress field}

It is assumed that the material parameters are homogeneous in the aggregate. The stress field in the polycrystal can be computed if the fields $J$, $\boldsymbol{D}, \boldsymbol{Q}$, and $\tau^{\mathrm{C}}$ are known. In the following we adopt the Voigt-Taylor assumption that the polycrystal deforms homogeneously on the microscale: $\bar{J}=J, \overline{\boldsymbol{D}}=\boldsymbol{D}$. Within this upper bound approximation, macroscopic anisotropies are due to an inhomogeneous distribution of the crystal orientations $\boldsymbol{Q} \in \mathrm{SO}(3)$ and the hardening state $\tau^{\mathrm{C}} \in \mathbb{R}^{+}$.

The state of the polycrystal will be described by a distribution function $h\left(\boldsymbol{Q}, \tau^{C}\right)$

$\frac{\mathrm{d} V}{V}\left(\boldsymbol{Q}, \tau^{\mathrm{C}}\right)=h\left(\boldsymbol{Q}, \tau^{\mathrm{C}}\right) \mathrm{d} Q \mathrm{~d} \tau^{\mathrm{C}}$,

which specifies the volume fraction of crystals having the orientation $\boldsymbol{Q}$ and the drag stress $\tau^{\mathrm{C}}$. The distribution function $h\left(\boldsymbol{Q}, \tau^{\mathrm{C}}\right)$ is non-negative

$h\left(\boldsymbol{Q}, \tau^{\mathrm{C}}\right) \geqslant 0 \quad \forall \boldsymbol{Q} \in \operatorname{SO}(3), \tau^{\mathrm{C}} \in \mathbb{R}^{+}$ and normalized in the sense that

$\int_{\mathscr{H}} h\left(\boldsymbol{Q}, \tau^{\mathrm{C}}\right) \mathrm{d} Q \mathrm{~d} \tau^{\mathrm{C}}=1$,

where $\mathscr{H}=\mathbb{R}^{+} \times \mathrm{SO}(3)$. If $\mathrm{SO}(3)$ is parameterized by Euler angles, the volume element $\mathrm{d} Q$ is given by

$\mathrm{d} Q=\frac{\sin (\Phi)}{8 \pi^{2}} \mathrm{~d} \varphi_{1} \mathrm{~d} \Phi \mathrm{d} \varphi_{2}$.

The codf $f(\boldsymbol{Q})$ is obtained by integrating $h\left(\boldsymbol{Q}, \tau^{\mathrm{C}}\right)$ over $\mathbb{R}^{+}$

$f(\boldsymbol{Q})=\int_{\mathbb{R}^{+}} h\left(\boldsymbol{Q}, \tau^{\mathrm{C}}\right) \mathrm{d} \tau^{\mathrm{C}}$.

For ergodic sample sets, the ensemble average of the stress tensor is given by the average of the Kirchhoff stress tensor over the reference volume $V$ of an arbitrary sample. Based on the Taylor assumption and by means of the distribution function $h$ the macroscopic stress tensor can be computed by

$\bar{\tau}=\frac{1}{V} \int_{V}\left(\tau^{\circ}+\tau^{\prime}\right) \mathrm{d} V=\bar{\tau}^{\circ}+\bar{\tau}^{\prime}$,

where

$\bar{\tau}^{\circ}=\bar{J} \frac{\partial W(\bar{J})}{\partial \bar{J}} \boldsymbol{I}=\frac{K}{2}\left(J^{2}-1\right) \boldsymbol{I}$

and

$\bar{\tau}^{\prime}=\int_{\mathscr{H}} h\left(\boldsymbol{Q}, \tau^{\mathrm{C}}\right) \tau^{\prime}\left(\overline{\boldsymbol{D}}^{\prime}, \boldsymbol{Q}, \tau^{\mathrm{C}}\right) \mathrm{d} Q \mathrm{~d} \tau^{\mathrm{C}}$.

If the hardening state is homogeneous in the aggregate, i.e. $h\left(\boldsymbol{Q}, \tau^{C}\right) \sim f(\boldsymbol{Q}) \delta_{\tau^{c}}$, then the last equation can be simplified to an orientational average of the stress tensor

$\bar{\tau}^{\prime}=\int_{\mathrm{SO}(3)} f(\boldsymbol{Q}) \tau^{\prime}\left(\overline{\boldsymbol{D}}^{\prime}, \boldsymbol{Q}, \tau^{\mathrm{C}}\right) \mathrm{d} Q$.

\subsection{Texture components}

Crystallographic textures can often be described by a small number of texture components or fibers [27,5,14]. A texture component is a crystal orientation for which the codf shows a (local) maximum in the elementary region. In the neighborhood, the codf is decreasing in an isotropic or anisotropic 
way. In the present work we exclusively consider texture components.

The distribution function $h\left(\boldsymbol{Q}, \tau^{\mathrm{C}}\right)$ is decomposed into an isotropic part $h^{\mathrm{I}}$ and an anisotropic part $h^{\mathrm{A}}$ with volume fractions $v^{\mathrm{I}}$ and $v^{\mathrm{A}}$, respectively. The anisotropic part is modeled as a superposition of $N_{\mathrm{c}}$ central distributions $g\left(\boldsymbol{Q}, \boldsymbol{Q}_{\alpha}, b_{\alpha}\right)$ with mean orientations $\boldsymbol{Q}_{\alpha}$ and volume fractions $v_{\alpha}^{\mathrm{A}}\left(\alpha=1, \ldots, N_{\mathrm{c}}\right)$. The hardening state of each of the components is assumed to be homogeneous and hence can be specified in terms of one drag stress $\tau_{\alpha}^{\mathrm{C}}$. The isotropic part depends on the arithmetic mean of the drag stresses $\bar{\tau}^{\mathrm{C}}=\sum_{\alpha=1}^{N_{\mathrm{c}}} v_{\alpha}^{\mathrm{A}} \tau_{\alpha}^{\mathrm{C}}$. As a result, we have

$h\left(\boldsymbol{Q}, \tau^{\mathrm{C}}\right)=v^{\mathrm{I}} h^{\mathrm{I}}\left(\bar{\tau}^{\mathrm{C}}\right)+v^{\mathrm{A}} h^{\mathrm{A}}\left(\boldsymbol{Q}, \boldsymbol{Q}_{\alpha}, b_{\alpha}, \tau_{\alpha}^{\mathrm{C}}\right)$,

where

$h^{\mathrm{I}}\left(\bar{\tau}^{\mathrm{C}}\right) \sim \delta_{\bar{\tau}^{\mathrm{C}}}$

and

$h^{\mathrm{A}}\left(\boldsymbol{Q}, \boldsymbol{Q}_{\alpha}, b_{\alpha}, \tau_{\alpha}^{\mathrm{C}}\right) \sim \sum_{\alpha=1}^{N_{\mathrm{c}}} v_{\alpha}^{\mathrm{A}} g\left(\boldsymbol{Q}, \boldsymbol{Q}_{\alpha}, b_{\alpha}\right) \delta_{\tau_{\alpha}^{\mathrm{C}}}$.

The following constraints upon the volume fractions hold:

$v^{\mathrm{I}}+v^{\mathrm{A}}=1, \quad \sum_{\alpha=1}^{N_{\mathrm{c}}} v_{\alpha}^{\mathrm{A}}=1$.

The value of a central distribution $g\left(\boldsymbol{Q}, \boldsymbol{Q}_{\alpha}, b_{\alpha}\right)$ at $\boldsymbol{Q}$ depends only on the distance $\omega$ between $\boldsymbol{Q}$ and $\boldsymbol{Q}_{\alpha}$, which is generally given by

$\omega\left(\boldsymbol{Q}, \boldsymbol{Q}_{\alpha}\right)=\arccos \left(\frac{1}{2}\left(\operatorname{tr}\left(\boldsymbol{Q} \boldsymbol{Q}_{\alpha}^{-1}\right)-1\right)\right)$

[5]. A specific central distribution is the MisesFischer distribution

$g\left(\boldsymbol{Q}, \boldsymbol{Q}_{\alpha}, b_{\alpha}\right)=N\left(S_{\alpha}\right) \mathrm{e}^{\left(S_{\alpha} \cos \left(\omega\left(\boldsymbol{Q}, \boldsymbol{Q}_{\alpha}\right)\right)\right)}$,

where

$N_{\alpha}=\frac{1}{I_{0}\left(S_{\alpha}\right)-I_{1}\left(S_{\alpha}\right)}$

and

$S_{\alpha}=S\left(b_{\alpha}\right)=\frac{\ln (2)}{2 \sin ^{2}\left(b_{\alpha} / 4\right)}$.
The parameter $b$ represents the half-width of the distribution. The modified Bessel functions $I_{n}$ are defined by

$I_{n}(S)=\frac{1}{\pi} \int_{0}^{\pi} \exp (S \cos (t)) \cos (n t) \mathrm{d} t$.

The Mises-Fischer distribution has the maximum entropy of all orientation distributions on SO(3) with expectation value of $\boldsymbol{Q}$ equal to $\boldsymbol{Q}_{\alpha}$. This distribution function was introduced by Mises in a two-dimensional case and by Fischer in a three-dimensional case [17].

Matthies [18] was the first one who applied the Mises-Fischer distribution in texture analysis. Matties called it normal distribution in the orientation space (see also [19]). The interpretation as a normal distribution was criticized by Schaeben $[23,24]$. An overview on central and noncentral distribution functions on $\mathrm{SO}(3)$ can be found in the monography by Mardia and Jupp [17]. Eschner $[7,8]$ used noncentral distribution functions for the description of experimental crystallographic textures.

A distribution function $h$ reflects both the symmetry of the crystallites forming the aggregate and the sample symmetry, which results from the processing history [29]. The crystal symmetry implies the following symmetry relation

$h\left(\boldsymbol{Q}, \tau^{\mathrm{C}}\right)=h\left(\boldsymbol{Q} \boldsymbol{H}^{\mathrm{C}}, \tau^{\mathrm{C}}\right)$

$\forall \boldsymbol{H}^{\mathrm{C}} \in S^{\mathrm{C}} \subseteq \mathrm{SO}(3) . S^{\mathrm{C}}$ denotes the symmetry group of the single crystal, which is assumed here to have a cubic symmetry. The sample symmetry implies the following symmetry relation

$h\left(\boldsymbol{Q}, \tau^{\mathrm{C}}\right)=h\left(\boldsymbol{H}^{\mathrm{S}} \boldsymbol{Q}, \tau^{\mathrm{C}}\right)$

$\forall \boldsymbol{H}^{\mathrm{S}} \in S^{\mathrm{S}} \subseteq \mathrm{SO}(3)$. $S^{\mathrm{S}}$ denotes the symmetry group of the sample. The following modified MisesFischer distribution implies the fulfillment of the constraint (30) upon the function $h$

$g\left(\boldsymbol{Q}, \boldsymbol{Q}_{\alpha}, b_{\alpha}\right)=\frac{1}{24} \sum_{\beta=1}^{24} N_{\alpha} \mathrm{e}^{S_{\alpha} \cos \left(\omega\left(\boldsymbol{Q}, \boldsymbol{H}_{\beta}^{\mathrm{C}} \boldsymbol{Q}_{\alpha}\right)\right.}$,

where the $\boldsymbol{H}_{\beta}^{\mathrm{C}} \in \mathrm{SO}(3)$ are the 24 elements of the symmetry group of cubic crystals.

Up to now the anisotropic part of the stress deviator is given by the orientational average 
$\bar{\tau}^{\prime \mathrm{A}}=\int_{\mathrm{SO}(3)} \sum_{\alpha=1}^{N_{\mathrm{c}}} v^{\mathrm{A}} v_{\alpha}^{\mathrm{A}} g\left(\boldsymbol{Q}, \boldsymbol{Q}_{\alpha}, b_{\alpha}\right) \tau^{\prime}\left(\overline{\boldsymbol{D}}^{\prime}, \boldsymbol{Q}, \tau_{\alpha}^{\mathrm{C}}\right) \mathrm{d} Q$.

After defining $\xi_{\alpha}$ by $\tau_{\alpha}^{\mathrm{C}}=\xi_{\alpha} \bar{\tau}^{\mathrm{C}}$ and taking into account that the stress deviator is homogeneous of degree $1 / m$ in the rate of deformation, it is possible to reformulate Eq. (33) by

$\bar{\tau}^{\prime \mathrm{A}}=\int_{\mathrm{SO}(3)} f^{\mathrm{A}}(\boldsymbol{Q}) \tau^{\prime}\left(\overline{\boldsymbol{D}}^{\prime}, \boldsymbol{Q}, \bar{\tau}^{\mathrm{C}}\right) \mathrm{d} Q$,

where

$f^{\mathrm{A}}(\boldsymbol{Q})=\sum_{\alpha=1}^{N_{\mathrm{c}}} \hat{v}_{\alpha}^{\mathrm{A}} g\left(\boldsymbol{Q}, \boldsymbol{Q}_{\alpha}, b_{\alpha}\right)$

and $\hat{v}^{\mathrm{A}}=v^{\mathrm{A}} v_{\alpha}^{\mathrm{A}} \xi^{m}$. It can be seen that an inhomogeneous hardening state affects the volume fractions that occur in the orientation distribution function. Since the stress deviator is implicitly given, the last equation makes the numerical determination of the stress tensor simpler. For a given $\overline{\boldsymbol{D}}^{\prime}$ the implicit equation has to be solved only once at $\bar{\tau}^{\mathrm{C}}$.

\section{Numerical examples}

Example 1. (Identification of the model based on experimental texture data) Lege et al. [15] determined the volume fractions, the half-widths, and the Euler angles of four texture components, which together with a random component, reproduce the main features of a crystallographic texture in a rolled aluminum sheet (see Table 1). The corresponding (100) pole figure is given in Fig. 1. Beside the crystallographic texture, the $R$

Table 1

Main texture components of a 2008-T4 sheet described by volume fractions, half widths and Euler angles on the condition that orthotropic sample symmetry is assumed

\begin{tabular}{llllll}
\hline$i$ & $b_{i}\left(^{\circ}\right)$ & $v_{i}$ & $\varphi_{1}^{i}$ & $\Phi^{i}$ & $\varphi_{2}^{i}$ \\
\hline 1 & 20.18 & 0.248 & 1.5532 & 1.5532 & 6.2656 \\
2 & 19.15 & 0.298 & 0.2564 & 1.4347 & 5.7036 \\
3 & 22.05 & 0.153 & 0.4664 & 1.5334 & 6.0412 \\
4 & 11.72 & 0.038 & 1.5549 & 1.5523 & 5.9513 \\
Random & & 0.263 & & & \\
\hline
\end{tabular}

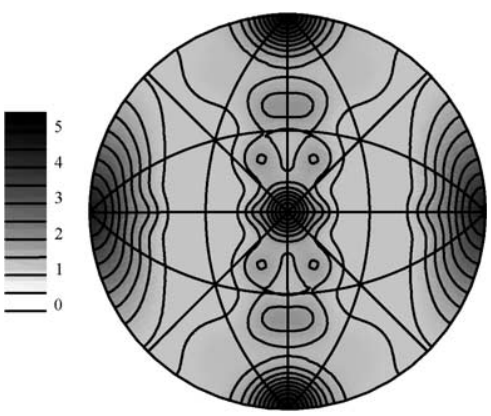

Fig. 1. (100) pole figure of a 2008-T4 sheet.

values and the yield stresses were also experimentally determined by tensile tests.

The numerical example presented here shows (i) how the identification of a phenomenological model can be avoided in the context of a viscoplastic modeling, and (ii) that the predictions based on the Taylor model can be obtained by the texture component model with much less degrees of freedom.

For the numerical simulation of the tensile test the codf is modeled by a superposition of four Mises-Fischer distributions and a random component, the parameters of which are given in Table 1. The half-width parameters are slightly different to the values determined by Lege et al. which is due to the application of different model functions for the components. Here, Mises-Fischer distribution functions are applied, whereas Lege et al. used pseudo-Gaussian distribution functions (see, e.g. [5]). The orthotropic sample symmetry of the polycrystal requires to use 16 components, which can be calculated by the components given in Table 1 and the symmetry transformations of the orthorhombic symmetry group.

The simulation of a tensile test with the texture component model requires the iterative search of a mean deviatoric strain rate which ensures a uniaxial overall stress state. The computation of the integrals in Eqs. (33) and (34) has been done numerically by a multidimensional adaptive integration algorithm for $n$-dimensional hyper-rectangles [1,2]. The $R$ value is determined by

$R=\frac{\overline{\boldsymbol{D}} \cdot \boldsymbol{n}_{\mathrm{w}} \otimes \boldsymbol{n}_{\mathrm{w}}}{\overline{\boldsymbol{D}} \cdot \boldsymbol{n}_{\mathrm{t}} \otimes \boldsymbol{n}_{\mathrm{t}}}$ 

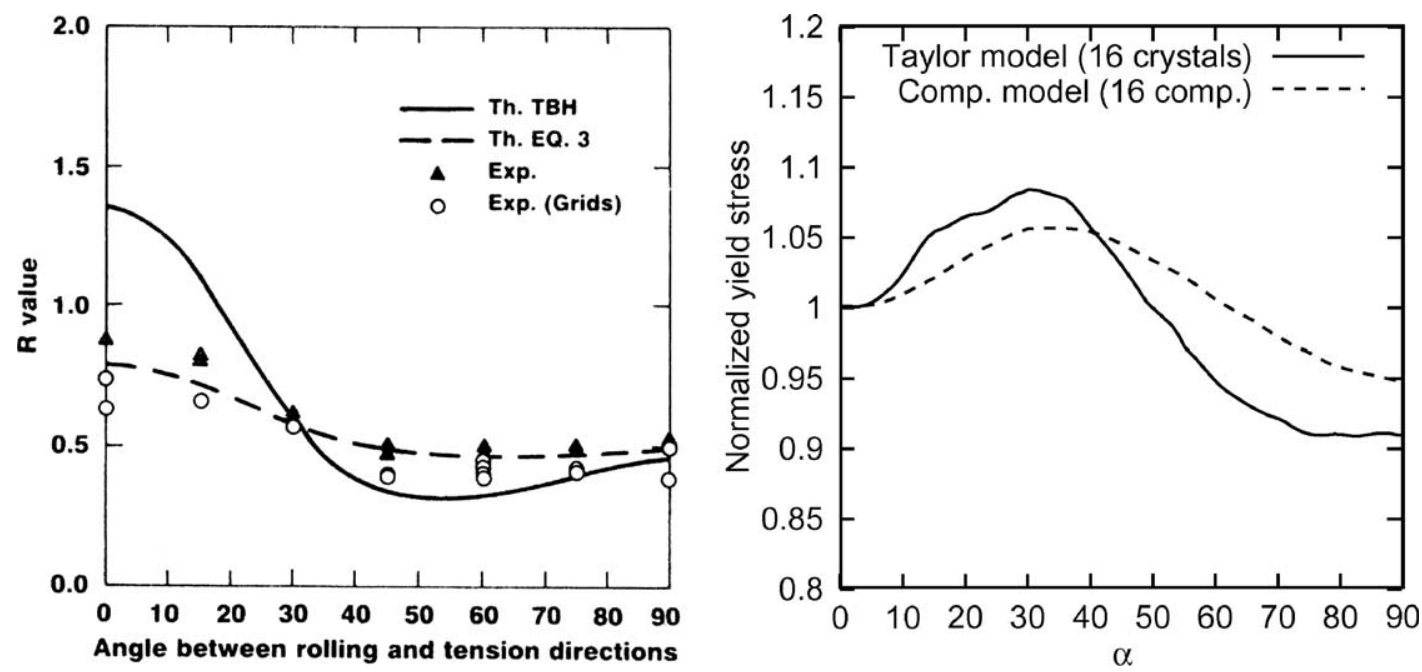

Fig. 2. Normalized yield stress versus tensile direction. Left: Numerical and experimental results taken from [15]. Right: Prediction of the texture component model and the Taylor model, respectively.

[14], where $\boldsymbol{n}_{\mathrm{w}}$ and $\boldsymbol{n}_{\mathrm{t}}$ define the width direction and the height direction, respectively. Fig. 2 (left) shows the yield stresses determined experimentally and numerically by Lege et al. Fig. 2 (right) shows the predictions of the texture component model, which are similar to the results obtained by the Taylor model based on several hundred crystal orientations. In contrast to the classical Taylor model the component model is based only on 16 different crystal orientations. Fig. 2 (right) also shows the prediction of the Taylor model based on 16 single crystal orientations. It can be seen that the component model predicts a much less pronounced anisotropy compared to the Taylor model (Fig. 3). This is due to the smoothing caused by Mises-Fischer distributions, which are used as weight functions for the stresses.

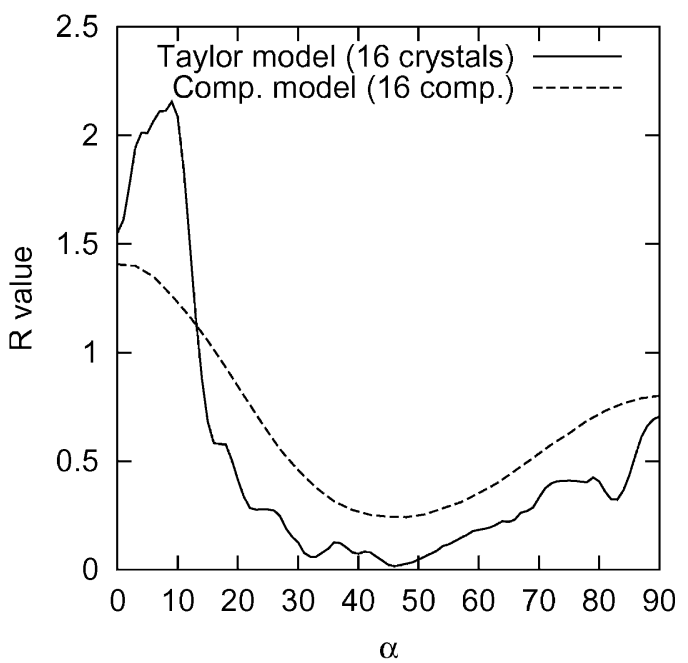

Fig. 3. $R$ value versus tensile direction. Left: Numerical and experimental results taken from [15]. Right: Prediction of the texture component model and the Taylor model, respectively. 

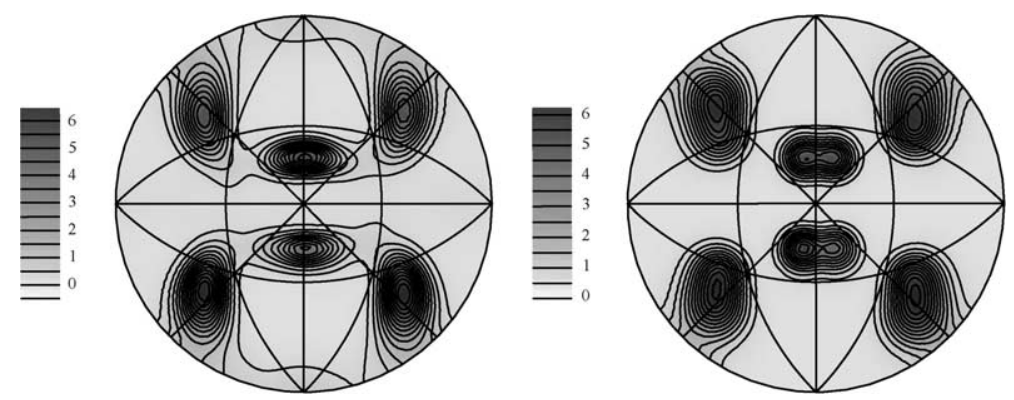

Fig. 4. (100) Pole figure of a rolling texture predicted by the Taylor model. Left: Classical Taylor model based on 1000 single crystal orientations, right: texture component model with four components.

Example 2. (Ideal rolling texture) Fig. 4 (left) shows the $(100)$ pole figure predicted by the Taylor model for a plane strain deformation mode with a final thickness reduction of $90 \%$. The dominant texture component that occurs in this texture is the Taylor orientation $\{4411\}\langle 11118\rangle$. It occurs two times due to the orthorhombic sample symmetry induced by the rolling deformation. The texture simulation has been performed with 1000 single crystals. The final codf $f_{1000}^{\mathrm{MF}}(\boldsymbol{Q})$ has been estimated by a superposition of 1000 MisesFischer distributions, one for each single crystal, with a half-width of $15^{\circ}$.

Fig. 4 (right) shows the (100) pole figure of the codf $f_{4}^{\mathrm{MF}}$ obtained by four Mises-Fischer distributions. The volume fractions and half-widths of the four distributions have been obtained by minimizing the distance $\delta$

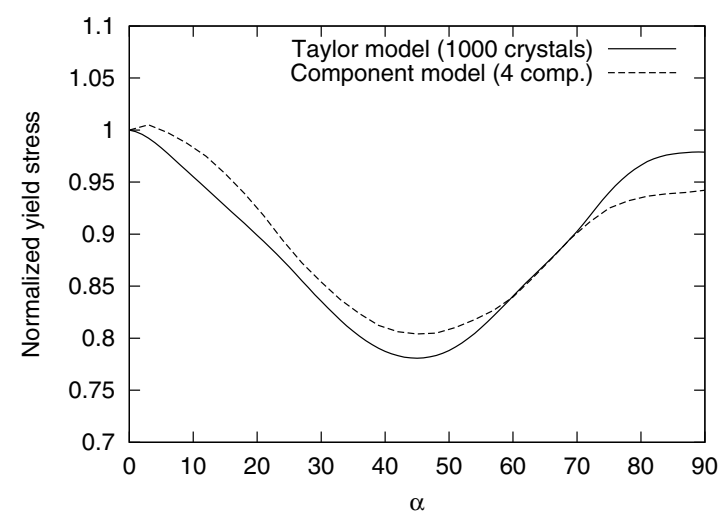

$\delta=\int_{\mathrm{SO}(3)}\left(f_{1000}^{\mathrm{MF}}(\boldsymbol{Q})-f_{4}^{\mathrm{MF}}(\boldsymbol{Q})\right)^{2} \mathrm{~d} Q$

between the $f_{1000}^{\mathrm{MF}}(\boldsymbol{Q})$ and $f_{4}^{\mathrm{MF}}(\boldsymbol{Q})$. The parameters, which are given in Table 2, have been obtained by an optimization scheme $[3,6]$.

Table 2

Half-width, volume fraction, and Euler angles, respectively, for the four Mises-Fischer components approximating the Taylor type texture shown in Fig. 4

\begin{tabular}{lllrrr}
\hline$i$ & $b_{i}\left(^{\circ}\right)$ & $v_{i}$ & \multicolumn{1}{c}{$\varphi_{1}^{i}$} & \multicolumn{1}{c}{$\Phi^{i}$} & \multicolumn{1}{c}{$\varphi_{2}^{i}$} \\
\hline 1 & 17.8 & 0.25 & 1.1740 & 0.5070 & -0.4942 \\
2 & 17.8 & 0.25 & -1.3009 & -0.4910 & 0.5855 \\
3 & 17.9 & 0.27 & 1.2762 & -0.4931 & -0.5678 \\
4 & 18.5 & 0.23 & -1.1476 & 0.5120 & 0.4743 \\
\hline
\end{tabular}

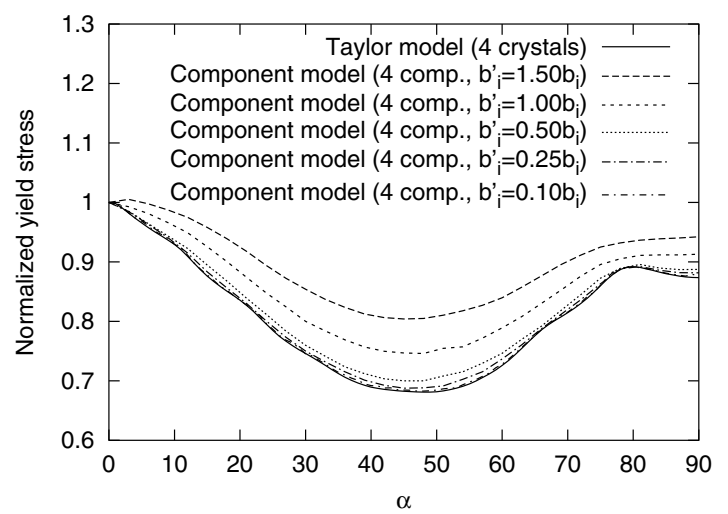

Fig. 5. Yield stress versus tensile direction. Left: Taylor model and component model. Right: Component model with different values of $b$. 

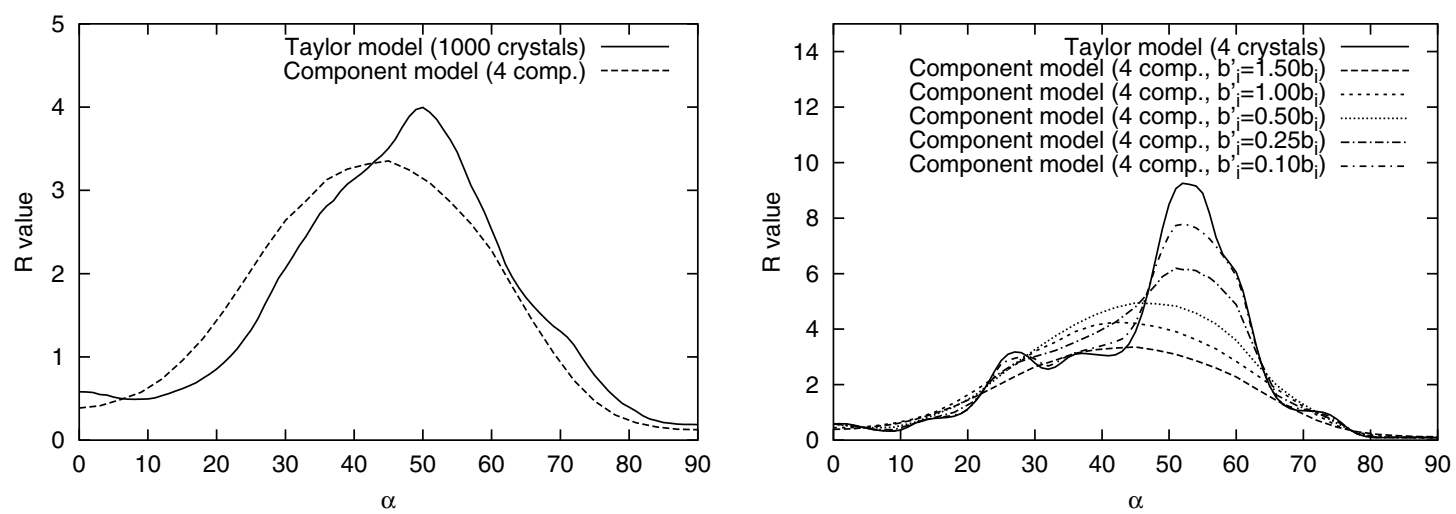

Fig. 6. $R$ value versus tensile direction. Left: Taylor model and component model. Right: Component model with different values of $b$.

For both textures the yield stresses and $R$ values were calculated using the Taylor and the component model, respectively. Fig. 5 (left) and Fig. 6 (left) show that the yield stresses and $R$ values predicted by the Taylor model and the component model are similar. In Fig. 5 (right) and in Fig. 6 (right) it can be seen that a Taylor simulation based on only four crystal orientations drastically over predicts the anisotropy. Furthermore, the results show that for vanishing half-widths the results of the component model approach the ones by the Taylor model.

\section{Summary}

In the present work we compared the classical Taylor model with a texture component model. The first represents the codf by a set of discrete single crystal orientations whereas the latter uses model functions which take into account the scattering of crystal orientations around the center of a texture component. It was shown that the application of square integrable distribution functions allows to reduce the number of single crystal orientations drastically.

\section{Acknowledgment}

Partial support for this research was provided by the Deutsche Forschungsgemeinschaft (DFG) under grant BE 1455/7 and GK 828.

\section{References}

[1] J. Berntsen, T. Espelid, An adaptive algorithm for the approximate calculation of multiple integrals, ACM Trans. Math. Software 17 (4) (1991) 437-451.

[2] J. Berntsen, T. Espelid, Algorithm 698: DCUHRE: an adaptive multidimensional integration routine for a vector of integrals, ACM Trans. Math. Software 17 (4) (1991) 452-456.

[3] C. Boender, A. Rinnooy Kan, G. Timmer, L. Stougie, A stochastic method for global optimization, Math. Program. 22 (1982) 125-140.

[4] E.A. Brandes, G.B. Brook, Smithells Metals Reference Book, seventh ed., Butterworth-Heinemann, Oxford, 1998.

[5] H.-J. Bunge, Texture Analysis in Material Science, Cuviller Verlag, Göttingen, 1993.

[6] T. Csendes, B.Z.H. Daroczy, Nonlinear parameter estimation by global optimization: comparison of local search methods in respiratory system modelling, Syst. Model. Optim. (1986) 188-192.

[7] T. Eschner, Texture analysis by means of model functions, Textures Microstruct. 21 (1993) 139-146.

[8] T. Eschner, Quantitative Texturanalyse durch Komponentenzerlegung von Beugungspolfiguren. Laborbericht PTB-7.4-95-1, Physikalisch-Technische Bundesanstalt, Braunschweig und Berlin, Abteilung Neutronenphysik, 1995.

[9] Y. Estrin, H. Mecking, A unified phenomenological description of work hardening and creep based on one parameter models, Acta Metall. (1984) 57-70. 
[10] K. Helming, Texturapproximation durch Modellkomponenten, Cuvillier Verlag, Göttingen, 1996.

[11] J. Hutchinson, Bounds and self-consistent estimates for creep of polycrystalline materials, Proc. R. Soc. Lond. A 348 (1976) 101-127.

[12] U. Kocks, Laws for work hardening and low temperature creep, J. Eng. Mater. Techn. (ASMEH) 98 (1976) $75-85$.

[13] U. Kocks, H. Mecking, Physics and phenomenology of strain hardening: the FCC case, Progr. Mater. Sci. 48 (2003) 171-273.

[14] U. Kocks, C. Tome, H. Wenk, Texture and Anisotropy: Preferred Orientations in Polycrystals and Their Effect on Material Properties, Cambridge University Press, Cambridge, 1998.

[15] D. Lege, F. Barlat, J. Brem, Characterization and modelling of the mechanical behavior and formability of a 2008T4 sheet sample, Int. J. Mech. Sci. 31 (7) (1989) 549-563.

[16] P. Les, M. Zehetbauer, E. Rauch, I. Kopacz, Cold work hardening of $\mathrm{Al}$ from shear deformation up to plarge strains, Scr. Mater. 41 (5) (1999) 523-528.

[17] K. Mardia, P. Jupp, Directional Statistics, John Wiley and Sons, New York, 2000.

[18] S. Matthies, Standard functions in texture analysis, Phys. Status Solidii B 101 (1980) K111-K115.

[19] S. Matthies, J. Muller, G. Vinel, On the normal distribution in the orientation space, Textures Microstruct. 10 (1988) 77-96.

[20] H. Mecking, Work hardening of single-phase polycrystals, Encyclopedia of Materials: Science and Technology, Elsevier Science, Amsterdam, 2001, pp. 9785-9795.
[21] R. Ogden, Large deformation isotropic elasticity: on the correlation of theory and experiment for compressible rubberlike materials, Proc. R. Soc. Lond. A 328 (1972) 567-583.

[22] D. Raabe, P. Klose, B. Engl, K.-P. Imlau, F. Friedel, F. Roters, Concepts for integrating plastic anisotropy into metal forming simulations, Adv. Eng. Mater. 4 (4) (2002) 169-180.

[23] H. Schaeben, "Normal" orientation distribution, Textures Microstruct. 19 (1992) 197-202.

[24] H. Schaeben, Diskrete mathematische Methoden zur Berechnung und Interpretation von kristallographischen Orientierungsdichten, DGM Informationsgesellschaft $\mathrm{mbH}, 1994$.

[25] J. Simo, C. Miehe, Associative coupled thermoplasticity at finite stains: formulation, numerical analyses and implementation, Comput. Methods Appl. Mech. Eng. 98 (1992) 41-104.

[26] M. Śilhavý, The Mechanics and Thermodynamics of Continuous Media, Springer, Berlin, 1997.

[27] G. Wasserman, J. Grewen, Texturen metallischer Werkstoffe, Springer, 1962.

[28] Z. Zhao, F. Roters, W. Mao, D. Raabe, Introduction of a texture component crystal plasticity finite element method for anisotropy simulations, Adv. Eng. Mater. 3 (12) (2001) 984-990.

[29] Q.-S. Zheng, Y.-B. Fu, Orientation distribution functions for microstructures of heterogeneous materials: II Crystal distribution functions and irreducible tensors restricted by various material symmetries, Appl. Math. Mech. 22 (8) (2001) 885-903. 